\title{
Exploring Functional Candidate Genes for Genetic Association in German Patients with Pseudoexfoliation Syndrome and Pseudoexfoliation Glaucoma
}

\author{
Mandy Krumbiegel, ${ }^{1}$ Francesca Pasutto, ${ }^{1}$ Christian Y. Mardin, ${ }^{2}$ Nicole Weisschub, ${ }^{3}$ \\ Daniela Paoli, ${ }^{4}$ Eugen Gramer, ${ }^{5}$ Matthias Zenkel, ${ }^{2}$ Bernhard H. F. Weber, ${ }^{6}$ \\ Friedrich E. Kruse, ${ }^{2}$ Ursula Schlötzer-Schrebardt, ${ }^{2}$ and André Reis ${ }^{1}$
}

Purpose. Pseudoexfoliation (PEX) syndrome is a generalized elastic microfibrillopathy characterized by fibrillar deposits in intra- and extraocular tissues. Genetic and nongenetic factors are known to be involved in its etiopathogenesis. This study was focused on six functional candidate genes involved in PEX material deposition and the analysis of their potential association with PEX syndrome and PEX glaucoma (PEXG).

METHODs. Fifty single-nucleotide polymorphisms (SNPs) capturing $>95 \%$ of overall genetic variance observed in Europeans at loci for FBN1, LTBP2, MFAP2, TGM2, TGF-b1, and $C L U$ were genotyped in 333 unrelated PEX-affected and 342 healthy individuals of German origin, and a genetic association study was performed. To replicate the findings, two SNPs of the $C L U$ gene were genotyped in a further 328 unrelated German patients with PEX as well as in 209 Italian patients with PEX and 190 Italian control subjects.

RESULTS. Association with PEX was observed only for the SNP rs2279590 in intron 8 of the $C L U$ gene coding for clusterin (corrected $P=0.0347$, OR $=1.34$ ) in our first German cohort. Likewise, a frequent haplotype encompassing the associated risk allele showed nominally significant association. None of remaining SNPs or SNP haplotypes were associated with PEX. The association found was confirmed in a second German cohort $(P=0.0244)$ but not in the Italian cohort $(P=0.7173)$. In addition, the association with $C L U$ SNP rs2279590 was more significant in German patients with PEX syndrome than in those with PEXG.

Concuusions. Genetic variants in the gene encoding clusterin may represent a risk factor for PEX in German patients but not in Italian patients. Variants in FBN1, LTBP2, MFAP2, TGF-b1, and TGM2 do not play a major role in the etiology of PEX

From the ${ }^{1}$ Institute of Human Genetics and the ${ }^{2}$ Department of Ophthalmology, University of Erlangen-Nuremberg, Erlangen, Germany; the ${ }^{3}$ Molecular Genetics Laboratory, University Eye Hospital, Tübingen, Germany; ${ }^{4}$ Reparto di Oftalmologia, Azienda Ospedaliera di Monfalcone, Monfalcone, Italy; the ${ }^{5}$ University Eye Hospital, Würzburg, Germany; and the "Institute of Human Genetics, University of Regensburg, Regensburg, Germany.

Supported by Grant SFB 539 from the German Research Foundation.

Submitted for publication May 27, 2008; revised November 1, 2008; accepted April 6, 2009.

Disclosure: M. Krumbiegel, None; F. Pasutto, None; C.Y. Mardin, None; N. Weisschuh, None; D. Paoli, None; E. Gramer, None; M. Zenkel, None; B.H.F. Weber, None; F.E. Kruse, None; U. Schlötzer-Schrehardt, None; A. Reis, None

The publication costs of this article were defrayed in part by page charge payment. This article must therefore be marked "advertisement" in accordance with 18 U.S.C. $\$ 1734$ solely to indicate this fact

Corresponding author: André Reis, Institute of Human Genetics, University of Erlangen-Nuremberg, Schwabachanlage 10, 91054 Erlangen, Germany; reis@humgenet.uni-erlangen.de. syndrome, at least in German patients. (Invest Ophthalmol Vis Sci. 2009;50:2796-2801) DOI:10.1167/iovs.08-2339

$\mathrm{P}$ seudoexfoliation (PEX) syndrome represents a common age-related systemic disorder affecting approximately $10 \%$ to $20 \%$ of the general population over age 60 and involving both genetic and nongenetic factors in its etiopathogenesis., It is characterized by pathologic accumulation of an abnormal fibrillar extracellular material in the anterior segment of the eye and in various extraocular tissues. ${ }^{3}$ The characteristic PEX fibrils appear to be multifocally produced by various intraocular cell types including the pre-equatorial lens epithelium, nonpigmented ciliary epithelium, trabecular endothelium, corneal endothelium, vascular endothelial cells, and virtually all cell types of the iris. ${ }^{4}$ The gradual buildup of PEX material in the trabecular meshwork most probably generates an increased outflow resistance and correlates with the intraocular pressure (IOP) level and the severity of glaucomatous optic nerve damage. ${ }^{5,6}$ PEX syndrome in fact is currently the most important single identifiable risk factor for open-angle glaucoma worldwide. ${ }^{7}$ In some populations (e.g., Baltic, Mediterranean, and Arabian), the frequency of PEX-associated secondary openangle glaucoma (PEXG) may reach a higher percentage than the primary form of the disease (POAG). For instance, PEX syndrome accounts for $77 \%$ of all cases of open-angle glaucoma in the eastern region of the Arabian Peninsula. ${ }^{8}$

Variable presence of PEX in different populations and increased risk of PEX in relatives of affected patients support a genetic basis for PEX. Recently, strong genetic association with single-nucleotide polymorphisms (SNPs) in the gene encoding lysyl oxidase-like 1 (LOXL1) was reported in patients from Iceland and Sweden.' Subsequently, this association has been widely replicated in other European and Asian populations, including our own study group. ${ }^{10}$

Although the underlying pathophysiology has not yet been elucidated, recent molecular biological evidence obtained from differential gene expression analyses suggests that PEX syndrome is an elastic microfibrillopathy associated with an excessive production of elastic microfibril components, such as fibrillin-1 (FBN1), microfibrillar-associated protein 2 (MFAP2), and latent transforming growth factor $\beta$-binding proteins (LTBPs), with enzymatic cross-linking processes involving transglutaminase (TGM2), increased levels of transforming growth factor (TGF-b1), and decreased levels of clusterin (CLU), an extracellular chaperone, that may promote the abnormal aggregation and deposition of the fibrillar material. ${ }^{11,12}$ A recent proteomic approach confirmed the presence of elastic fiber components and clusterin within PEX material deposits. ${ }^{13}$ Based on these findings, we evaluated, whether variations in functional candidate genes encoding fibrillin 1 (FBN1), latent transforming growth factor $\beta$-binding protein 2 (LTBP2), microfibrillar-associated protein 2 (MFAP2), transforming growth factor beta 1 (TGF-b1), transglutaminase 2 (TGM2), and clusterin $(C L U)$ are associated with PEX syndrome and PEXG. 
Table 1. Phenotypic Composition, Age and Sex Distribution in Patients with PEX or PEXG or and Healthy Subjects

\begin{tabular}{lcccccrrr}
\hline & $\boldsymbol{n}$ & PEX & PEXG & Mean Age \pm SD & Min. & Max. & Female & Male \\
\hline $\begin{array}{l}\text { German cohorts } \\
\quad \text { Patients }\end{array}$ & & & & & & & & \\
$\quad$ Discovery & 333 & 167 & 166 & $79.2 \pm 8.5$ & 49 & 99 & 195 \\
$\quad \begin{array}{l}\text { Replication } \\
\quad \text { Control subjects }\end{array}$ & 328 & 35 & 293 & $73.9 \pm 9.0$ & 51 & 98 & 192 \\
$\begin{array}{l}\text { Italian cohorts } \\
\quad \text { Patients }\end{array}$ & 209 & 76 & 133 & $73.9 \pm 6.4$ & 51 & 92 & 193 \\
$\quad$ Control subjects & 190 & & & $76.3 \pm 7.7$ & 60 & 101 & 149 \\
\hline
\end{tabular}

TABLE 2. Allele Frequencies of the 50 htSNPs in Patients and Control Subjects and Results of $\chi^{2}$ Statistics

\begin{tabular}{|c|c|c|c|c|c|c|c|}
\hline Gene/No. & Name & Allele & Cases & Controls & $\chi^{2}$ & $\boldsymbol{P}$ & $P c^{*}$ \\
\hline \multicolumn{8}{|l|}{ LTBP2 2} \\
\hline 1 & rs10149538 & G & 0.508 & 0.480 & 1.057 & 0.3040 & \\
\hline 2 & rs 2190876 & A & 0.461 & 0.434 & 0.989 & 0.3199 & \\
\hline 3 & rs11621693 & A & 0.153 & 0.146 & 0.140 & 0.7081 & \\
\hline 4 & rs 10146812 & $\mathrm{~A}$ & 0.721 & 0.670 & 4.160 & 0.0414 & 0.4222 \\
\hline 5 & rs 1005154 & $\mathrm{C}$ & 0.394 & 0.349 & 2.899 & 0.0886 & \\
\hline 6 & rs862046 & C & 0.597 & 0.519 & 7.928 & 0.0049 & 0.0733 \\
\hline 7 & rs2302114 & G & 0.580 & 0.519 & 5.081 & 0.0242 & 0.2837 \\
\hline 8 & rs699370 & A & 0.423 & 0.354 & 6.571 & 0.0104 & 0.1400 \\
\hline 9 & $\mathrm{rs} 3815328$ & G & 0.663 & 0.653 & 0.144 & 0.7047 & \\
\hline 10 & rs862026 & $\mathrm{C}$ & 0.432 & 0.406 & 0.905 & 0.3414 & \\
\hline 11 & rs 12588574 & G & 0.795 & 0.769 & 1.287 & 0.2566 & \\
\hline 12 & rs11159088 & G & 0.412 & 0.374 & 1.931 & 0.1646 & \\
\hline 13 & rs7148764 & $\mathrm{T}$ & 0.524 & 0.497 & 0.938 & 0.3328 & \\
\hline 14 & rs7150659 & $\mathrm{C}$ & 0.592 & 0.542 & 3.260 & 0.0710 & \\
\hline 15 & rs 4903242 & $\mathrm{~T}$ & 0.541 & 0.519 & 0.633 & 0.4263 & \\
\hline 16 & rs3784024 & $\mathrm{T}$ & 0.785 & 0.779 & 0.087 & 0.7685 & \\
\hline 17 & rs7401961 & G & 0.636 & 0.617 & 0.499 & 0.4798 & \\
\hline 18 & rs 7150223 & $\mathrm{~T}$ & 0.488 & 0.471 & 0.401 & 0.5264 & \\
\hline 19 & rs8014087 & $\mathrm{C}$ & 0.541 & 0.526 & 0.288 & 0.5912 & \\
\hline 20 & rs 12435481 & $\mathrm{C}$ & 0.566 & 0.560 & 0.052 & 0.8197 & \\
\hline 21 & rs8006778 & $\mathrm{T}$ & 0.495 & 0.465 & 1.246 & 0.2643 & \\
\hline 22 & rs1866628 & $\mathrm{T}$ & 0.498 & 0.450 & 3.085 & 0.0790 & \\
\hline 23 & rs989910 & C & 0.502 & 0.466 & 1.639 & 0.2004 & \\
\hline 24 & rs11159094 & A & 0.479 & 0.447 & 1.341 & 0.2468 & \\
\hline 25 & rs1077662 & G & 0.469 & 0.455 & 0.280 & 0.5964 & \\
\hline 26 & rs888414 & $\mathrm{A}$ & 0.391 & 0.377 & 0.268 & 0.6050 & \\
\hline 27 & rs12892228 & $\mathrm{T}$ & 0.472 & 0.468 & 0.027 & 0.8700 & \\
\hline 28 & rs 10047892 & $\mathrm{~T}$ & 0.539 & 0.525 & 0.273 & 0.6015 & \\
\hline 29 & rs11621186 & $\mathrm{T}$ & 0.524 & 0.509 & 0.293 & 0.5883 & \\
\hline \multicolumn{8}{|l|}{$C L U$} \\
\hline 1 & rs3087554 & A & 0.828 & 0.796 & 2.232 & 0.1352 & \\
\hline 2 & rs2279590 & A & 0.417 & 0.347 & 6.882 & 0.0087 & 0.0347 \\
\hline 3 & rs9331931 & $\mathrm{C}$ & 0.748 & 0.719 & 1.386 & 0.2390 & \\
\hline 4 & rs11136000 & A & 0.398 & 0.340 & 4.838 & 0.0278 & 0.0958 \\
\hline \multirow{2}{*}{\multicolumn{8}{|c|}{ FBN1 }} \\
\hline & & & & & & & \\
\hline 1 & rs1042078 & $\mathrm{C}$ & 0.288 & 0.243 & 3.481 & 0.0621 & \\
\hline 2 & rs2291117 & $\mathrm{C}$ & 0.122 & 0.119 & 0.026 & 0.8720 & \\
\hline 3 & rs1807301 & G & 0.252 & 0.227 & 1.154 & 0.2827 & \\
\hline \multicolumn{8}{|l|}{ MFAP2 } \\
\hline 1 & rs6691985 & $\mathrm{C}$ & 0.721 & 0.673 & 3.315 & 0.0686 & \\
\hline 2 & rs12097163 & G & 0.228 & 0.212 & 0.489 & 0.4842 & \\
\hline 3 & rs3754511 & $\mathrm{T}$ & 0.811 & 0.796 & 0.452 & 0.5014 & \\
\hline 4 & rs3738815 & G & 0.818 & 0.783 & 2.470 & 0.1160 & \\
\hline 5 & rs2076604 & A & 0.821 & 0.783 & 3.140 & 0.0764 & \\
\hline \multicolumn{8}{|l|}{$T G F-b 1$} \\
\hline 1 & rs8179181 & $\mathrm{T}$ & 0.272 & 0.259 & 0.293 & 0.5885 & \\
\hline 2 & rs 4803455 & A & 0.502 & 0.499 & 0.012 & 0.9119 & \\
\hline \multirow{2}{*}{\multicolumn{8}{|c|}{ TGM2 }} \\
\hline & & & & & & & \\
\hline 1 & rs6127200 & $\mathrm{C}$ & 0.161 & 0.158 & 0.024 & 0.8775 & \\
\hline 2 & rs11696730 & $\mathrm{T}$ & 0.942 & 0.925 & 1.676 & 0.1954 & \\
\hline 3 & rs6023527 & G & 0.161 & 0.119 & 4.771 & 0.0289 & 0.1329 \\
\hline 4 & rs 4811529 & G & 0.150 & 0.118 & 2.926 & 0.0872 & \\
\hline 5 & rs2235582 & $\mathrm{A}$ & 0.411 & 0.361 & 3.560 & 0.0592 & \\
\hline
\end{tabular}

\footnotetext{
${ }^{*} P$ c value: $P$ after permutation correction $(n=10,000)$.
} 


\section{Methods}

\section{Study Populations}

The study was approved by the ethics review boards of the Medical Faculty of the University of Erlangen-Nuremberg (Germany) and that of the hospital in Monfalcone-Gorizia (Italy) and was conducted in accordance with the tenets of the Declaration of Helsinki. All subjects gave informed consent before entering the study. The first group of patients with PEX (discovery group) consisted of a total of 333, the replication group of 328 subjects of German origin. All individuals underwent standardized clinical examination for signs of PEX syndrome at the Ophthalmology Department of the University of Erlangen-Nuremberg and Würzburg. All patients with PEX had to have manifest PEX material on the anterior capsule and/or pupillary margin in mydriasis by slit lamp biomicroscopy. Secondary open-angle glaucoma due to PEX syndrome was defined, if elevated intraocular pressure (IOP $>20 \mathrm{~mm} \mathrm{Hg}$ ), an open chamber angle, characteristic visual field defects in computed perimetry, and characteristic glaucomatous disc atrophy were found in the presence of manifest PEX deposits on the anterior lens capsule and/or pupillary margin. Age and sex distribution of the groups of patients are shown in Table 1. Two hundred nine patients of Italian origin were examined at the Ophthalmology Department of the Monfalcone Hospital (Italy) with identical clinical examinations.

A total of 342 healthy German subjects and 190 healthy Italian subjects were recruited from the same geographic regions as the patients. All control subjects underwent ophthalmic examination and were matched for age and sex (Table 1). Overall, healthy individuals had IOP levels below $20 \mathrm{~mm} \mathrm{Hg}$, no glaucomatous disc damage, no PEX material deposits on anterior lens capsule and/or pupillary margin, no clinical signs indicating early or suspect PEX (e.g., atrophy of the iridal pigment epithelium at the pupillary margin, secondary melanin dispersion in the chamber angle and anterior chamber after dilation of the pupil, no dewlike condensation on the anterior lens capsule, normal mydriasis) and no family history of PEX or glaucoma. Visual acuity was at least 0.8 and the optic media were clear for ophthalmic examination.

\section{DNA Extraction and Genotyping}

Genomic DNAs were extracted from peripheral blood leukocytes of patients with PEX and control individuals by using automated techniques (Flex 3000; AutoGen Holliston, MA, using Flexigene chemistry; Qiagen, Hilden, Germany). Fifty haplotype-tagging (ht)SNPs distributed among the six candidate genes (29 in $L T B P 2,5$ in $C L U, 5$ in MFAP2, 5 in TGM2, 3 in $F B N 1$, and 3 in TGF-b1) were selected capturing more than $95 \%$ of genetic diversity in Europeans, based on the HapMap CEPH (CEU) data (http://www.hapmap.org/). Genotyping of 40 htSNPs was performed with a genotyping system assay (SNPlex; Applied Biosystems [ABI], Foster City, CA). Reactions were prepared according to the manufacturer's protocol and analyzed on a DNA analyzer (model 3730; ABI). Data were evaluated with gene mapping software (GeneMapper Software ver. 4.0; ABI). Ten SNPs were genotyped with predeveloped assays (TaqMan; ABI). Reactions were prepared according to the manufacturer's instructions and analyzed on a sequence-detection system (Prism 7900HT; ABI), using standard thermal cycling conditions. Genotype data for four arbitrarily selected SNPs was verified by direct sequencing of 24 randomly chosen samples. The average genotyping rate was $98.5 \%$. Hardy-Weinberg equilibrium for all SNPs was confirmed in the control group with the program Haploview. ${ }^{14}$

\section{Statistical Analysis}

Association analysis used allele counts $\left(\chi^{2}\right)$. The haplotype block structure and SNP haplotype association was performed with the program Haploview which uses $\chi^{2}$ statistics for assessing haplotype association (version 4.0). ${ }^{14} P \leq 0.05$ was considered statistically significant. Obtained $P$ values were corrected for multiple testing by a permutation test (10,000 permutations; Haploview 4.0).

\section{Results}

Association analysis in the German discovery cohort (Table 1) was performed with the 29 SNPs identified in $L T B P 2,5$ in MFAP2, 5 in TGM2, 3 in FBN1, 3 in TGF-b1, and 5 in $C L U$. Among all SNPs analyzed, only allele A of SNP rs2279590 located at intron 8 of $C L U$ encoding for clusterin showed a marked difference in allelic frequency between patients with PEX and control subjects $(P=0.0087$; Table 2$)$. This association remained significant after correction for multiple testing with permutation analysis (corrected $P=0.0347$, OR $=1.34$; Table 2). Haplotypes constructed with the five $C L U$ SNPs were also examined. Of interest the most frequent haplotype encompassing the associated risk allele A of the SNP rs2279590 showed nominally significant association with $\operatorname{PEX}(P=0.05$, Fig. 1$)$.

Recently, a strong association of variants in lysyl oxidaselike 1 ( $L O X L 1$ ) in PEX and PEXG was reported. ${ }^{9}$ Therefore, we compared the allele frequencies at $C L U$ locus conditioned on the presence of the identified LOXL1 common risk haplotype G-G, which is composed of the major alleles $G$ of the two

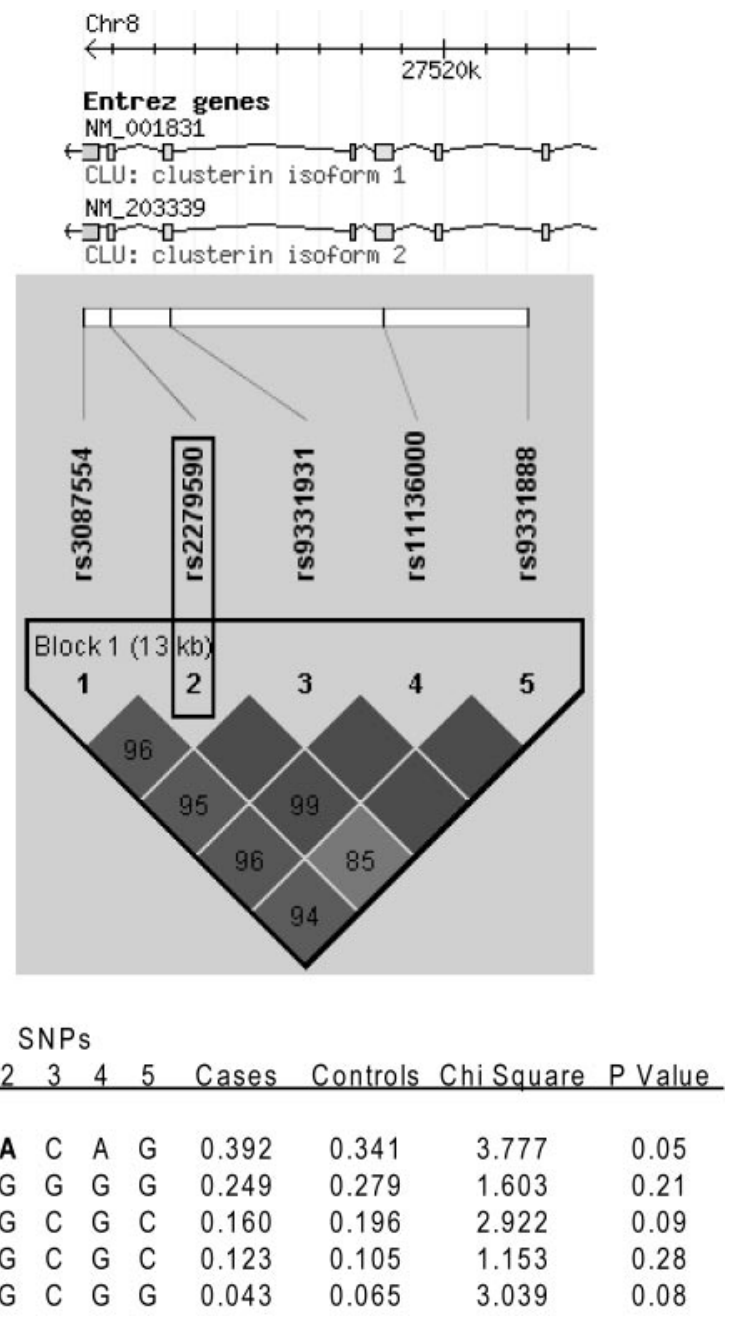

FigURE 1. LD structure of the genomic region of $C L U$ and derived haplotypes with corresponding frequencies in our cohorts. Haploview plot showing pair-wise LD based on genotypes of 333 patients and 342 controls. Dark diamonds: regions of high LD $\left(\mathrm{D}^{\prime}>0.8\right)$. The relative chromosomal position of each SNP in relation to the genomic organization of the clusterin gene is given in the upper diagram. Bottom: distribution of respective haplotypes between patients and controls. Haplotype 1 carrying the associated risk allele at SNP 2 (rs2279590, bold) shows nominally significant association. 
TABLE 3. Association of $C L U$ SNP rs2279590 Allele A with $L O X L 1$ Common Risk Haplotype G-G, Composed of Both G Alleles of the Two LOXL1 Coding SNPs rs1048661 and rs3825942

\begin{tabular}{|c|c|c|c|c|c|c|c|c|c|}
\hline $\begin{array}{c}\text { LOXL1 } \\
\text { Haplotypes }\end{array}$ & $\begin{array}{l}\text { Patients } \\
\text { (n) }\end{array}$ & $\begin{array}{c}\text { Controls } \\
\text { (n) }\end{array}$ & Case & Control & $\chi^{2}$ & $\boldsymbol{P}$ & $\boldsymbol{P} \boldsymbol{c}^{*}$ & OR & $95 \% \mathrm{CI}$ \\
\hline G-G carriers & 307 & 254 & 0.420 & 0.338 & 7.757 & 0.0054 & 0.0062 & 1.416 & $1.1082-1.8103$ \\
\hline Non-G-G carriers & 26 & 88 & 0.385 & 0.374 & 0.021 & 0.8852 & 1.0000 & & \\
\hline
\end{tabular}

${ }^{*} P \mathrm{c}, P$ after permutation correction $(n=10,000)$.

coding SNPs rs1048661 and rs3825942. We stratified our discovery German cohort and control group for carriers and noncarriers of the risk haplotype G-G (Table 3). Although we observed no association of the subgroups of non G-G carriers, association to allele A of $C L U$ SNP rs2279590 in carriers of LOXL1 G-G haplotype was significant (corrected $P=0.0062$, Table 3 ).

To replicate the observed association, we genotyped SNP rs2279590 tagging the associated $C L U$ haplotype, A-A-C-A-G (Fig. 1), in the replication cohort of 328 German patients with PEX (Table 1). When comparing this cohort with the ophthalmologically examined and age-matched control group, we found a similar difference in allelic frequency between patients with PEX and control subjects $(P=0.0244)$. To more faithfully assess the strength of the associated risk, we combined both German PEX cohorts (Table 4). A significant association was observed in all patients with PEX only for allele A of SNP rs2279590 ( $P=0.0050$, corrected $P=0.0123)$. Of note, this association was more significant in patients classified with PEX syndrome than with PEXG. The associated risk, though, is rather modest $(\mathrm{OR}=1.45$, Table 4$)$.

In addition, we genotyped SNP rs3087554 recently reported by Burdon et al. ${ }^{15}$ as possibly being associated with PEX syndrome in the Australian Blue Mountains Eye Study cohort. This SNP was not associated with PEX in the German combined cohort; however, after the subclassification allele A of this SNP was nominally significant associated with PEX syndrome (Table 4). A second replication cohort of 209 patients with PEX collected in Northern Italy was also genotyped for both SNPs, rs2279590 and rs3087554, but showed no evidence of association, either at single SNP or at haplotype levels (Tables 4, 5).

In the combined German cohort (661 patients), though, haplotype analysis of both SNPs, which are in complete linkage disequilibrium (LD, $\mathrm{D}^{\prime} \sim 1$, Fig. 1), showed haplotype AA to be overrepresented compared with the control group. This association results also more significant in patients with PEX syndrome than in patients with PEXG (Table 5).

\section{Discussion}

One currently proposed pathogenic concept of PEX syndrome describes the condition as a specific type of stress-induced elastosis, an elastic microfibrillopathy, associated with the excessive production of elastic microfibrils and their aggregation into mature PEX fibrils by a variety of potentially elastogenic cells. ${ }^{16}$ Growth factors, particularly TGF- $\beta 1$, increased cellular and oxidative stress, and an impaired cellular protection and proteasome system appear to be key factors in its pathogenesis. ${ }^{17}$ Because of an imbalance between matrix metalloproteinases and their inhibitors and extensive cross-linking processes involved in fiber formation, the pathologic material is not properly degraded but progressively accumulates in tissues (e.g., trabecular meshwork), over time. ${ }^{18}$ This concept of a generalized elastosis gained substantial support by a recently published genetic study showing that polymorphisms in the lysyl oxidase-like 1 (LOXL1) gene, which is involved in elastic fiber formation and stabilization, are associated with PEX syndrome and PEX glaucoma.'

Five of the candidate genes analyzed in this study, FBN1, $L T B P 2, M F A P 2, T G M 2$, and $T G F-b 1$, are related to extracellular matrix metabolism and showed increased expression levels in anterior segment tissues of patients with PEX compared with ocular tissues of healthy individuals. ${ }^{12}$ Hence, we hypothesized that genetic differences in these genes could underlie these differential expression patterns and confer risk to PEX. However, this approach failed to find any association of PEX with any of the single 45 examined SNPs or with the here from derived haplotypes. In fact, no significant differences in allele or haplotype distribution in these five candidate genes were detected between patients with PEX and control individuals, suggesting that genetic variants at these loci are not relevant in the development of PEX syndrome, at least in German patients.

Nevertheless, our study identified an association between an intronic SNP in $C L U$, rs2279590, with PEX syndrome. The association was seen in both the discovery and replication cohorts from Germany suggesting that genetic variation at this

TABLE 4. ORs of $C L U$ SNPs rs2279590 and rs3087554 in PEX and PEXG from Germany and Italy

\begin{tabular}{|c|c|c|c|c|c|c|c|}
\hline \multirow[b]{2}{*}{ Study Population (n) } & \multicolumn{4}{|c|}{ rs2279590 A } & \multicolumn{3}{|c|}{ rs3087554 A } \\
\hline & Freq. & OR $(95 \%$ CI) & $\boldsymbol{P}$ & $P c$ & Freq. & OR $(95 \% \mathrm{CI})$ & $\boldsymbol{P}$ \\
\hline \multicolumn{8}{|l|}{ Germany } \\
\hline Controls (342) & 0.347 & & & & 0.796 & & \\
\hline PEX combined (661) & 0.412 & $1.32(1.09-1.60)$ & 0.0050 & 0.0123 & 0.828 & $1.23(0.97-1.56)$ & 0.0816 \\
\hline PEXG (459) & 0.402 & $1.26(1.03-1.55)$ & 0.0260 & & 0.817 & $1.14(0.89-1.47)$ & 0.2964 \\
\hline PEX (202) & 0.435 & $1.45(1.12-1.87)$ & 0.0041 & 0.0110 & 0.853 & $1.49(1.06-2.07)$ & 0.0195 \\
\hline \multicolumn{8}{|l|}{ Italy } \\
\hline Controls (190) & 0.429 & & & & 0.832 & & \\
\hline PEX combined (209) & 0.416 & $0.95(0.72-1.26)$ & 0.7173 & & 0.841 & $1.07(0.74-1.56)$ & 0.7096 \\
\hline PEXG (133) & 0.413 & $0.94(0.68-1.29)$ & 0.6920 & & 0.831 & $0.99(0.65-1.51)$ & 0.9800 \\
\hline PEX (76) & 0.421 & $0.97(0.66-1.42)$ & 0.8742 & & 0.860 & $1.24(0.73-2.12)$ & 0.4219 \\
\hline
\end{tabular}

${ }^{*} P$ c, $P$ after permutation correction $(n=10,000)$ 
TABLE 5. Association of PEX and PEXG with Haplotypes Formed by the Two CLU SNPs rs 3087554 and rs2279590 in German Patients

\begin{tabular}{lcccccc}
\hline & Haplotype & Cases & Controls & $\chi^{2}$ & $\boldsymbol{P}$ & Pc \\
\hline PEX combined & & $n=661$ & $n=342$ & & & \\
& AG & 0.419 & 0.451 & 1.905 & & \\
\multirow{4}{*}{ PEXG } & AA & 0.410 & 0.346 & 7.686 & 0.0056 & 0.0130 \\
& GG & 0.169 & 0.201 & 3.025 & & \\
PEX & AG & $n=459$ & $n=342$ & & & \\
& AA & 0.418 & 0.451 & 1.783 & & \\
& GG & 0.400 & 0.346 & 4.877 & 0.0272 & \\
& AG & $n=202$ & $n=342$ & 1.047 & & \\
& AA & 0.422 & 0.451 & 0.904 & & \\
& GG & 0.431 & 0.346 & 7.935 & 0.0048 & 0.0138 \\
\hline
\end{tabular}

$P c, P$ after permutation correction $(n=10,000)$.

locus is indeed involved in the etiopathogenesis of PEX. In contrast, no association was evident in the Italian study group. Several factors such as population specific differences in disease susceptibility loci or insufficient statistical power could explain this difference. Of note, the association in the German cohorts was more prevalent in patients with PEX without glaucoma than in patients with PEXG. This finding could be interpreted as variants in $C L U$ predisposing primarily to the pathologic PEX process itself rather than to the glaucomatous process. The risk conferred, though, is modest with an OR around 1.5 , as is typical for many disease susceptibility variants identified in complex diseases.

Given the relative small effect size of the observed association, though, it is still possible that our finding is due to a type I error, despite the large sample size of our cohort. Thus, we assessed the significance of our finding with 10,000 random permutation tests. When correcting $P$ values in this way, the association at SNP rs2279590 remained significant, suggesting that this is a true finding. Of interest, the association is only seen in patients with PEX carrying the LOXL1-risk haplotype G-G but not in the small group carrying other nonrisk haplotypes. Whether this is an incidental finding or due to a true (genetic) interaction of either genes or their products remains to be determined. At present, there are no data available concerning an interaction between clusterin and any other of the candidate genes analyzed with $L O X L 1$.

Previous studies demonstrated a significantly reduced expression of the extracellular chaperone clusterin in the anterior segment of PEX eyes. ${ }^{11,12}$ In vitro, clusterin has been found to inhibit the formation of insoluble amyloid fibrils resulting from the aggregation of amyloid $\beta$, apolipoprotein C-II, and a variety of other fibril-forming peptides. ${ }^{19,20}$ Clusterin also has been reported to associate with soluble amyloid $\beta$ in plasma and cerebrospinal fluid, suggesting that the interaction may preclude amyloid $\beta$ aggregation and fibrillization in biological fluids. ${ }^{19,20}$ This observed deficiency in clusterin, a highly efficient extracellular chaperone, may therefore promote the chronic, stable accumulation of PEX material, an abnormal fibrillar extracellular matrix product, in anterior segment tissues of PEX eyes.

Clusterin gene expression appears to be responsive to a variety of cytokines, growth factors, and stress-inducing agents through many regulatory elements within the $C L U$ promoter region. ${ }^{21}$ Although it is very well possible that SNP rs2279590 is only in $\mathrm{LD}$ with another causative variant in $C L U$, we cannot exclude that it itself has an effect on gene expression, which led us to perform a bioinformatic analysis of intron 8 for transcription factor binding sites (MatInspector software; Genomatix, Munich, Germany). ${ }^{22}$ Of interest, this program predicts a potential intronic vitamin $\mathrm{D}$ receptor responsive element (VDRE) for the allele containing the major nonrisk allele $\mathrm{G}$ of rs2279590. This hypothesis must be corroborated by future functional studies, to provide conclusive evidence of its potential causality. Similarly, investigations of this SNP in other populations, especially in patients with PEX without glaucoma are now needed to further strengthen the association of $C L U$ with PEX syndrome.

\section{Acknowledgments}

The authors thank all the patients and control individuals who participated in this study, Juliane Niedziella for invaluable help with patient recruitment, and Claudia Preller for expert technical assistance.

\section{References}

1. Forsius H, Forsman E, Fellman J, Eriksson AW. Exfoliation syndrome: frequency, gender distribution and association with climatically induced alterations of the cornea and conjunctiva. Acta Opbthalmol Scand. 2002;80:478-484.

2. Ringvold A. Epidemiology of the pseudo-exfoliation syndrome. Acta Opbthalmol Scand. 1999;77:371-375.

3. Ritch R, Schlotzer-Schrehardt U. Exfoliation syndrome. Surv Ophthalmol. 2001;45:265-315.

4. Naumann GO, Schlotzer-Schrehardt U, Kuchle M. Pseudoexfoliation syndrome for the comprehensive ophthalmologist. Intraocular and systemic manifestations. Opbthalmology. 1998;105:951968.

5. Gottanka J, Flugel-Koch C, Martus P, Johnson DH, Lütjen-Drecoll E. Correlation of pseudoexfoliative material and optic nerve damage in pseudoexfoliation syndrome. Invest Ophthalmol Vis Sci. 1997; 38:2435-2446.

6. Schlotzer-Schrehardt U, Naumann GO. Trabecular meshwork in pseudoexfoliation syndrome with and without open-angle glaucoma: a morphometric, ultrastructural study. Invest Ophthalmol Vis Sci. 1995;36:1750-1764.

7. Ritch R, Schlotzer-Schrehardt U, Konstas AG. Why is glaucoma associated with exfoliation syndrome? Prog Retin Eye Res. 2003; 22:253-275.

8. Bialasiewicz AA, Wali U, Shenoy R, Al-Saeidi R. Patients with secondary open-angle glaucoma in pseudoexfoliation (PEX) syndrome among a population with high prevalence of PEX. Clinical findings and morphological and surgical characteristics (in German). Ophthalmologe. 2005;102:1064-1068.

9. Thorleifsson G, Magnusson KP, Sulem P, et al. Common sequence variants in the LOXL1 gene confer susceptibility to exfoliation glaucoma. Science. 2007;317:1397-1400.

10. Pasutto F, Krumbiegel M, Mardin CY, et al. Association of LOXL1 Common sequence variants in German and Italian patients with pseudoexfoliation syndrome and pseudoexfoliation glaucoma. Invest Ophthalmol Vis Sci. 2008;49:1459-1463. 
11. Zenkel M, Kruse FE, Junemann AG, Naumann GO, Schlotzer-Schrehardt U. Clusterin deficiency in eyes with pseudoexfoliation syndrome may be implicated in the aggregation and deposition of pseudoexfoliative material. Invest Opbthalmol Vis Sci. 2006;47: 1982-1990.

12. Zenkel M, Poschl E, von der Mark K, et al. Differential gene expression in pseudoexfoliation syndrome. Invest Ophthalmol Vis Sci. 2005;46:3742-3752.

13. Ovodenko B, Rostagno A, Neubert TA, et al. Proteomic analysis of exfoliation deposits. Invest Opbthalmol Vis Sci. 2007;48:14471457.

14. Barrett JC, Fry B, Maller J, Daly MJ. Haploview: analysis and visualization of LD and haplotype maps. Bioinformatics. 2005;21:263265.

15. Burdon KP, Sharma S, Hewitt AW, et al. Genetic analysis of the clusterin gene in pseudoexfoliation syndrome. Mol Vis. 2008;14: 1727-1736.

16. Schlotzer-Schrehardt U, Naumann GO. Ocular and systemic pseudoexfoliation syndrome. Am J Opbthalmol. 2006;141:921937.
17. Zenkel M, Kruse FE, Naumann GO, Schlotzer-Schrehardt U. Impaired cytoprotective mechanisms in eyes with pseudoexfoliation syndrome/glaucoma. Invest Ophthalmol Vis Sci. 2007;48:55585566.

18. Schlotzer-Schrehardt U, Lommatzsch J, Kuchle M, Konstas AG, Naumann GO. Matrix metalloproteinases and their inhibitors in aqueous humor of patients with pseudoexfoliation syndrome/ glaucoma and primary open-angle glaucoma. Invest Ophthalmol Vis Sci. 2003;44:1117-1125.

19. Calero M, Rostagno A, Frangione B, Ghiso J. Clusterin and Alzheimer's disease. Subcell Biochem. 2005;38:273-298.

20. Matsubara E, Soto C, Governale S, Frangione B, Ghiso J. Apolipoprotein J and Alzheimer's amyloid beta solubility. Biochem J. 1996;316:671-679.

21. Trougakos IP, Gonos ES. Clusterin/apolipoprotein J in human aging and cancer. Int J Biochem Cell Biol. 2002;34:1430-1448.

22. Quandt K, Frech K, Karas H, Wingender E, Werner T. MatInd and MatInspector: new fast and versatile tools for detection of consensus matches in nucleotide sequence data. Nucleic Acids Res. 1995; $23: 4878-4884$. 